\title{
Design and fabrication of double-chirped mirrors
}

\author{
F. X. Kärtner, N. Matuschek, T. Schibli, and U. Keller \\ Ultrafast Laser Physics Laboratory, Institute of Quantum Electronics, Swiss Federal Institute of Technology, ETH Hönggerberg-HPT, \\ CH-8093 Zürich, Switzerland

\section{H. A. Haus} \\ Department of Electrical Engineering and Computer Science, and Research Laboratory of Electronics, Massachusetts Institute of \\ Technology, Cambridge, Massachusetts 02139

\section{Heine and R. Morf} \\ Paul-Scherrer-Institute, Zürich, CH-8040 Zürich, Switzerland
}

\author{
V. Scheuer, M. Tilsch, and T. Tschudi \\ Institute for Applied Physics, TH Darmstadt, D-64289 Darmstadt, Germany
}

Received January 2, 1997

\begin{abstract}
We present an analytic design method for the reproducible fabrication of double-chirped mirrors to achieve simultaneously a high reflectivity and dispersion compensation over an extended bandwidth compared with those of standard quarter-wave Bragg mirrors. The mirrors are fabricated by ion beam sputtering. Use of these mirrors in a Ti:sapphire laser leads to 6.5 -fs pulses directly out of the laser. The method can also be applied to the design of chirped-fiber gratings and general optical filters. (c) 1997 Optical Society of America
\end{abstract}

Short-pulse generation has advanced to a degree where the bandwidth of standard Bragg mirrors composed of $\mathrm{SiO}_{2} / \mathrm{TiO}_{2}$ layer pairs limits the pulse width of ultrashort pulsed lasers. Chirped mirrors ${ }^{1}$ overcome the bandwidth limitation and provide simultaneously a means for compensation of the group-delay dispersion in the laser. However, so far the chirped-mirror design has been done by computer optimization, ${ }^{1}$ because a simple chirping of the center wavelength of the Bragg mirror is not enough. ${ }^{2}$ Chirping would introduce oscillations in the group delay for longer wavelengths, which penetrate deeper into the mirror. The oscillations are due to an effective Gire-Tournois interferometer. The interferometer is created by partial reflection from the front section of the mirror, which acts as a transmission grating, and the reflection at the back of the mirror, which acts as a Bragg reflector.

We present an analytic design for chirped mirrors based on coupled-mode and transmission-line theory. The cause of the oscillations in the group-delay dispersion is identified as an impedance-matching problem. We solve this problem by slowly tapering the impedance, which is shown to be equivalent to a chirp in the thickness of the high-index layer in addition to the chirp of the center wavelength of the Bragg mirror. Therefore, we call the resulting structure a doublechirped mirror.

We start from a coupled-mode description of a Bragg mirror composed of an alternating sequence of highand low-index layers as shown in Fig. 1. Recently, ${ }^{3}$ we showed that one can obtain the transfer matrix for a symmetric index step with high and low refractive indices $n_{h}, n_{l}$, respectively, and corresponding layer thicknesses $d_{h, m}, d_{l, m}$ correctly by solving the following coupled-mode equations over an interval of unit length:

$$
\begin{aligned}
& \frac{\mathrm{d} A}{\mathrm{~d} m}=-i \delta(m) A(m)-i \kappa(m) B(m), \\
& \frac{\mathrm{d} B}{\mathrm{~d} m}=+i \kappa(m) A(m)+i \delta(m) B(m) .
\end{aligned}
$$

Here, $A$ and $B$ denote the slowly varying amplitudes of the rightward- and leftward-propagating electromagnetic field, respectively, which differ from the original amplitudes only by an alternating factor $(-1)^{m}$ at $m \in \mathbb{N}_{0}$; but this factor is irrelevant for the computation of reflectivity and group delay. The coupled-mode equations (1) are exact for arbitrary index discontinuities if the proper, dimensionless detuning coefficient $\delta$ and coupling coefficient $\kappa$ are used, which are constant over the corresponding unit interval. Following

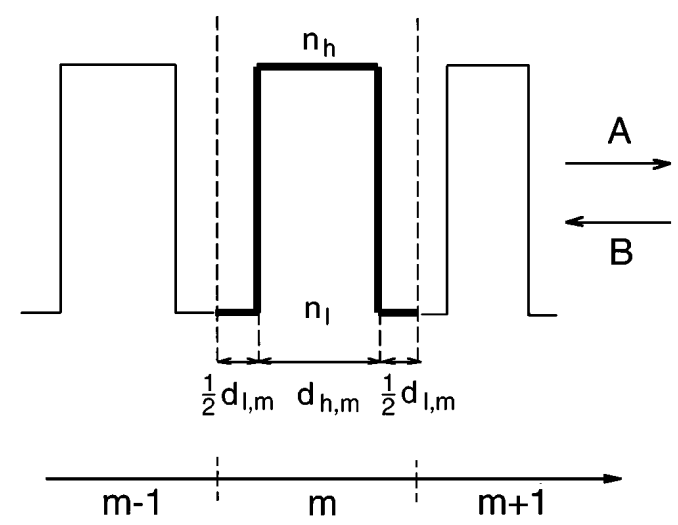

Fig. 1. A general Bragg structure can be decomposed into a series of symmetric index steps.

(C) 1997 Optical Society of America 
Ref. 3, the exact coefficients are given by

$$
\begin{aligned}
\delta(m)= & -\alpha(m) \frac{1}{1-r^{2}}\left\{\sin \left[\phi_{h}(m)+\phi_{l}(m)\right]\right. \\
& \left.+r^{2} \sin \left[\phi_{h}(m)-\phi_{l}(m)\right]\right\}, \\
\kappa(m)= & -\alpha(m) \frac{2 r}{1-r^{2}} \sin \left[\phi_{h}(m)\right],
\end{aligned}
$$

with

$$
\alpha(m)=\frac{\eta(m)}{\sinh [\eta(m)]},
$$

where $\eta(m)$ is uniquely defined by the conditions

$$
\cosh [\eta(m)]=-F_{R}(m), \quad \sinh [\eta(m)]=\left[F_{R}^{2}(m)-1\right]^{1 / 2},
$$

with

$$
\begin{aligned}
F_{R}(m)= & \frac{1}{1-r^{2}}\left\{\cos \left[\phi_{h}(m)+\phi_{l}(m)\right]\right. \\
& \left.-r^{2} \cos \left[\phi_{h}(m)-\phi_{l}(m)\right]\right\} .
\end{aligned}
$$

Here, $r=\left(n_{h}-n_{l}\right) /\left(n_{h}+n_{l}\right)$ denotes the Fresnel reflectivity at one index discontinuity and $\phi_{l}(m)=$ $k n_{l} d_{l, m}, \phi_{h}(m)=k n_{h} d_{h, m}$ are the phase shifts of the wave in the low- and high-index regions, respectively, of the $m$ th index step, where, $k$ is the vacuum wave number.

To get insight into the rather complex Eqs. (2) and (3), which are, however, correct for any case, we simplify the expressions for the coupling and detuning coefficients by retaining only terms of first order in $r \ll 1$. Under these conditions, the wave number at zero detuning, $k_{B, m}=\pi /\left(n_{l} d_{l, m}+n_{h} d_{h, m}\right)$, defines the center wave number or the Bragg wave number of a resulting Bragg mirror composed of a series of equal index steps. If the wave number $k$ is close to $k_{B}$ then $\alpha \approx 1$, and the detuning and coupling coefficients for the $m$ th index step can be approximated by $\delta(m) \approx \phi_{h}(m)+\phi_{l}(m)-\pi$ and $\kappa(m) \approx-2 r \sin \left[\phi_{h}(m)\right]$, which shows that both the detuning and the coupling coefficients of the equivalent coupled-mode equations can be independently designed by the thickness of the low- and high-index layers, respectively.

Coupled-mode equations (1) are equivalent to the following telegraph equations for the voltage $V=$ $(A+B) / \sqrt{2}$ and the current $I=(A-B) / \sqrt{2}$ of an artificial transmission line with reactance $X(m)$ and susceptance $Y(m)$ for each layer pair:

$$
\begin{aligned}
\mathrm{d} V / \mathrm{d} m=-i X(m) I, & X=\delta-\kappa, \\
\mathrm{d} I / \mathrm{d} m=-i Y(m) V, & Y=\delta+\kappa .
\end{aligned}
$$

The characteristic impedance of this transmission line is then given by $Z=\sqrt{X / Y}$. To avoid spurious reflections in an inhomogeneous transmission line, we know from microwave engineering ${ }^{4}$ that the characteristic impedance has to be changed slowly. One can find the same condition by using a WKB solution ${ }^{5}$ for the Schrödinger equation that results from elimination of the current or voltage from Eqs. (4). At $m=0$, that is, in front of the Bragg grating, we have no coupling between the forward- and the backward-traveling waves. Thus, in front of the first layer the characteristic impedance is 1. Equations (4) show that tapering of the characteristic impedance from 1 to a different value can be achieved simultaneously for all frequencies if the coupling coefficient in the coupledmode equations is adiabatically switched on. The coupling coefficient is proportional to the sine of the optical phase delay in the high-refractive-index layer. Thus, to avoid Fabry-Perot effects, one has to chirp not only the Bragg wavelength of the mirror but also the thickness of the high-index layer, which we refer to as a double-chirped mirror.

As an example, Fig. 2 shows the reflectivity and group delay of several Bragg mirrors composed of 25 index steps, with $n_{h}=2.5, n_{l}=1.5$, similar to the refractive indices of $\mathrm{TiO}_{2}$ and $\mathrm{SiO}_{2}$, which results in a Fresnel reflectivity $r=0.25$. In the first case (Fig. 2, dotted curves) only the Bragg wave number is linearly chirped from $6.8 \mu \mathrm{m}^{-1} \leq k_{B}=2 \pi / \lambda_{B} \leq$ $11 \mu \mathrm{m}^{-1}$ over the first 20 index steps and held constant over the last 5 index steps. The large oscillation in the group delay prevents the use of such mirrors in femtosecond lasers. ${ }^{2}$ The dashed and solid curves in Fig. 2 show the reflectivity and the group delay obtained with double-chirped mirrors, respectively; the thickness of the high-index layers is increased gradually over the first 12 index steps according to $d_{h, m}=$ $(m / 12)^{a} \lambda_{B, 12} /\left(4 n_{h}\right)$ with $\alpha=1,2$. We can clearly see the strong reduction of the oscillations in the group delay by using the double-chirp technique. Quadratic tapering of the coupling coefficient already eliminates

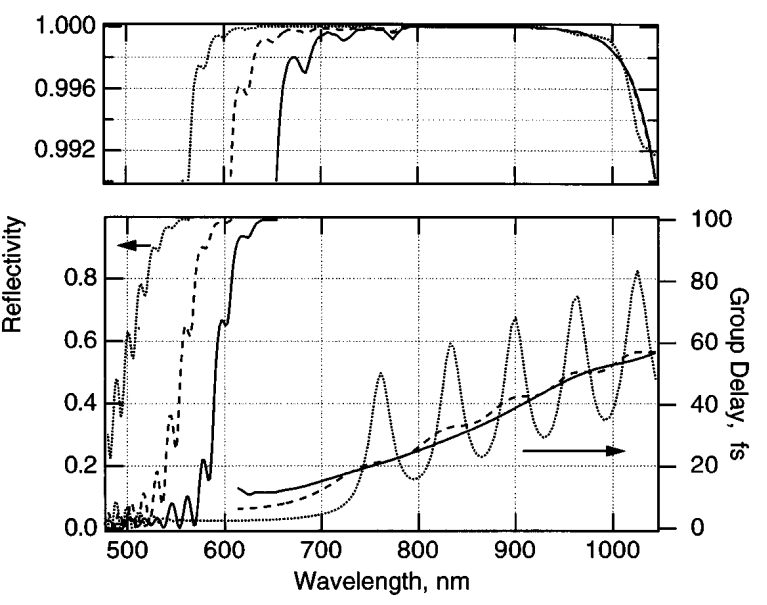

Fig. 2. Comparison of the reflectivity and the group delay of chirped mirrors with 25 layer pairs with refractive indices $n_{l}=1.5, n_{h}=2.5$. The upper portion shows the enlarged top percent of the reflectivity. The dotted curves show the result for a simply chirped mirror. The dashed and solid curves show the result for double-chirped mirrors, where in addition to the chirp in the Bragg wave number $k_{B}$ the thickness of the high-index layers is also chirped over the first 12 layer pairs from zero to its maximum value for a linear chirp (dashed curves) and for a quadratic chirp (solid curves). 


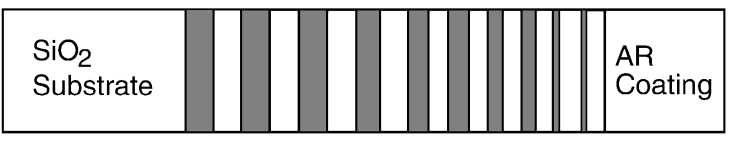

Fig. 3. Schematic picture of the universal structure of a double-chirped mirror. AR, antireflection.
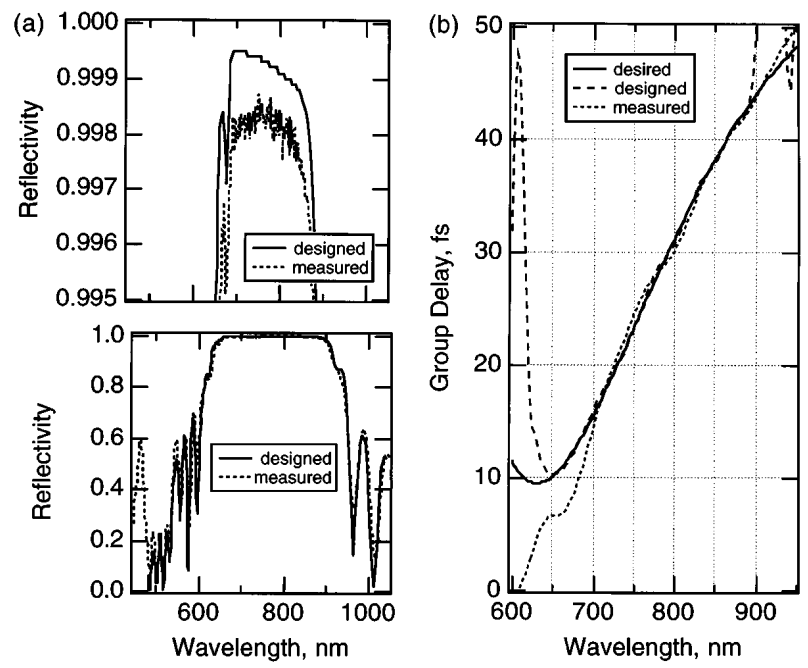

Fig. 4. Designed and measured (a) reflectivity and (b) group delay of the fabricated double-chirped mirror as described in the text.

the oscillations in the group delay. Because of the double chirp we automatically generate high transmission at the pump wavelength of the Ti:sapphire laser near $500 \mathrm{~nm}$.

So far, we have not considered the refractive-index jump from the top mirror layer to the air, which again would introduce a Gire-Tournois interferometer into the mirror. To avoid having this occur, we designed a broadband antiref lection coating separately by using a simulated thermal annealing technique. ${ }^{6}$ The antireflection coating matches the Bragg mirror to the air. Finally, the resulting mirror was optimized by a standard gradient method to minimize again the remaining oscillations in the group delay. The universal schematic structure of the resulting double-chirped mirror is shown in Fig. 3.

With the procedure described above we designed a mirror with the following properties: (a) reflectivity of $\sim 99.8 \%$ in the wavelength range $650-890 \mathrm{~nm}$ centered at $760 \mathrm{~nm}$, (b) high transmission at $500 \mathrm{~nm}$ for the argon-ion pump laser, and (c) a given negative and smooth group-delay dispersion in the high-reflectivity range. In total, we used 25 layer pairs, 5 for the antireflection coating and 20 for the double-chirped Bragg structure. Ion beam sputtering was used for the layer growth, ${ }^{7}$ and a precise layer control during growth was indispensable. ${ }^{8}$ Figures 4(a) and 4(b) show the designed and the measured reflectivity and group delay, respectively, of the fabricated mirror. Additionally, in Fig. 4(b) the initially desired group delay for compensating the higher-order dispersion of the Ti:sapphire laser ${ }^{9}$ is shown. Because of the limited number of layer pairs the desired group delay can be approached only over a limited bandwidth. Obviously, the properties of the designed and fabricated mirror are close to the design goal, which demonstrates the reproducible fabrication of this mirror. Because of the semianalytic design, which automatically avoids internal resonances in the mirror, and the high quality of the ion beam sputtered layers, reflectivities as large as $99.8 \%$ are reached despite the fact that most of the light penetrates deep into the mirror. According to Ref. 10, internal resonances might plague computer-optimized structures. Currently, the remaining oscillation in the group delay is due to the limited number of layer pairs. A Kerr-lens mode-locked Ti:sapphire laser with such a double-chirped mirror and a broadband semiconductor saturable-absorber mirror ${ }^{11,12}$ as a starting mechanism produced self-starting 6.5 -fs pulses directly from the laser. ${ }^{9}$

In conclusion, we have derived a method for the design of broadband, dispersion-compensating mirrors based on coupled-mode and transmission-line theory. The typical strong oscillations in the group delay shown by such structures are identified as an impedance-matching problem, which can be solved by an additional chirping of the coupling coefficient, which results in double-chirped mirrors. The ideas presented here can be applied to the design of multilayer interference coatings and filters in general and also to the design of chirped-fiber gratings.

This research was supported by the Swiss National Science Foundation and the German National Science Foundation.

\section{References}

1. R. Szipöcs, K. Ferencz, C. Spielmann, and F. Krausz, Opt. Lett. 19, 201 (1994).

2. P. Laporta and V. Magni, Appl. Opt. 24, 2014 (1985).

3. N. Matuschek, F. X. Kärtner, and U. Keller, IEEE J. Quantum Electron. 33, 295 (1997).

4. R. E. Collin, Foundations for Microwave Engineering, 2nd ed. (McGraw-Hill, New York, 1992), Chaps. 3 and 5.

5. A. B. Migdal, Qualitative Methods in Quantum Theory (Addison-Wesley, Reading, Mass., 1977), Chap. 3.

6. R. Morf and R. E. Kunz, Proc. SPIE 1270, 11 (1990).

7. V. Scheuer, M. Tilsch, and T. Tschudi, Proc. SPIE 2253, 445 (1994).

8. M. Tilsch, V. Scheuer, and T. Tschudi, Proc. SPIE 2253, 414 (1994).

9. I. D. Jung, F. X. Kärtner, N. Matuschek, D. H. Sutter, F. Morier-Genoud, G. Zhang, U. Keller, V. Scheuer, M. Tilsch, and T. Tschudi, "Self-starting 6.5-fs pulses from a Ti:sapphire laser," Opt. Lett. (to be published).

10. L. Xu, C. Spielmann, F. Krausz, and R. Szipöcs, Opt. Lett. 21, 1259 (1996).

11. R. Fluck, I. D. Jung, G. Zhang, F. X. Kärtner, and U. Keller, Opt. Lett. 21, 743 (1996).

12. U. Keller, K. J. Weingarten, F. X. Kärtner, D. Kopf, B. Braun, I. D. Jung, R. Fluck, C. Hönninger, N. Matuschek, and J. Aus der Au, "Semiconductor saturable absorber mirrors (SESAMs) for femtosecond to nanosecond pulse generation in solid-state lasers," J. Sel. Topics Quantum Electron. (to be published). 